\title{
Nuclear Energy Research Initiative Program (NERI) Quarterly Progress Report: New Design Equations for Swelling and Irradiation Creep in Generation IV Reactors
}

W. G. Wolfer, M. P. Surh, F. A. Garner, Jr., D. C. Chrzan, C. Schaldach, J. B. Sturgeon

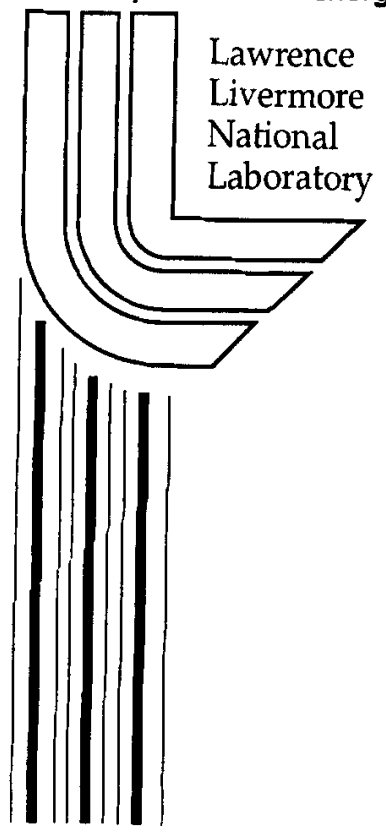

February 13, 2003 


\section{DISCLAIMER}

This document was prepared as an account of work sponsored by an agency of the United States Government. Neither the United States Government nor the University of California nor any of their employees, makes any warranty, express or implied, or assumes any legal liability or responsibility for the accuracy, completeness, or usefulness of any information, apparatus, product, or process disclosed, or represents that its use would not infringe privately owned rights. Reference herein to any specific commercial product, process, or service by trade name, trademark, manufacturer, or otherwise, does not necessarily constitute or imply its endorsement, recommendation, or favoring by the United States Government or the University of California. The views and opinions of authors expressed herein do not necessarily state or reflect those of the United States Government or the University of California, and shall not be used for advertising or product endorsement purposes.

This work was performed under the auspices of the U.S. Department of Energy by the University of California, Lawrence Livermore National Laboratory under Contract No. W-7405-Eng-48.

This report has been reproduced directly from the best available copy.

Available electronically at http://www.doc.gov/bridge

Available for a processing fee to U.S. Department of Energy

And its contractors in paper from

U.S. Department of Energy

Office of Scientific and Technical Information

P.O. Box 62

Oak Ridge, TN 37831-0062

Telephone: (865) 576-8401

Facsimile: (865) 576-5728

E-mail: reports@adonis.osti.gov

Available for the sale to the public from

U.S. Department of Commerce

National Technical Information Service

5285 Port Royal Road

Springfield, VA 22161

Telephone: (800) 553-6847

Facsimile: (703) 605-6900

E-mail: orders@ntis.fedworld.gov

Online ordering: http://www.ntis.gov/ordering.htm

OR

Lawrence Livermore National Laboratory

Technical Information Department's Digital Library

http://www.llnl.gov/tid/Library.html 
NUCLEAR ENERGY RESEARCH INITIATIVE PROGRAM (NERI)

QUARTERLY PROGRESS REPORT

\author{
New Design Equations for Swelling and Irradiation Creep \\ in Generation IV Reactors
}

Project Number: 01-137

First Annual Report

May - September 2002

\author{
Submitted by: \\ Lead Organization: Lawrence Livermore National Laboratory \\ Compiled by M.P. Surh
}

P.I.

Wilhelm G. Wolfer and Michael P. Surh

Lawrence Livermore National Laboratory, CA

Collaborators:

Frank A. Garner, Jr. Pacific Northwest National Laboratory, Richland, WA

Daryl C. Chrzan, University of California, Berkeley, CA

Charlene Schaldach, Jess B. Sturgeon, Lawrence Livermore National Laboratory, CA

Submitted on:

February 13, 2003 


\section{$\underline{\text { Research Obiectives }}$}

The objectives of this research project are to significantly extend the theoretical foundation and the modeling of radiation-induced microstructural changes in structural materials used in Generation $I V$ nuclear reactors, and to derive from these microstructure models the constitutive laws for void swelling, irradiation creep and stress-induced swelling, as well as changes in mechanical properties.

The need for the proposed research is based on three major developments and advances over the past two decades. First, new experimental discoveries have been made on void swelling and irradiation creep which invalidate previous theoretical models and empirical constitutive laws for swelling and irradiation creep. Second, recent advances in computational methods and power make it now possible to model the complex processes of microstructure evolution over long-term neutron exposures. Third, it is now required that radiation-induced changes in structural materials over extended lifetimes be predicted and incorporated in the design of Generation IV reactors.

Our approach to modeling and data analysis is a dual one in accord with both the objectives to simulate the evolution of the microstucture and to develop design equations for macroscopic properties. Validation of the models through data analysis is therefore carried out at both the microscopic and the macroscopic levels. For the microstructure models, we utilize the transmission electron microscopy results from steels irradiated in reactors and from model materials irradiated by neutrons as well as ion bombardments.

The macroscopic constitutive laws will be tested and validated by analyzing density data, irradiation creep data, diameter changes of fuel elements, and post-irradiation tensile data. Validation of both microstructure models and macroscopic constitutive laws is a more stringent test of the internal consistency of the underlying science for radiation effects in structural materials for nuclear reactors.

\section{$\underline{\text { Research Progress }}$}

This document relates progress for the first fiscal year of our project FY2002, primarily consisting of two quarters spanning the months of May to September 2002.

There are two first-year milestones listed in the proposal:

1) Complete void nucleation code with dislocation evolution included. Verify predicted incubation doses with data from solution-annealed and 20\% CW $316 \mathrm{SS}$.

2) Complete a separate code for dislocation loop formation and growth and verify predictions with microstructural data from pure $\mathrm{Fe}-15 \mathrm{Cr}-16 \mathrm{Ni}$ alloys.

The quarterly reports have emphasized 5 subtasks, including the institutional responsibilities:

- Void and Bubble Nucleation and Growth (LLNL)

- Dislocation Loop Formation and Network Evolution (LLNL)

- Detailed Dislocation-Void Co-Evolution (beyond mean field) (Berkeley, LLNL)

- Model Integration (complete-microstructure evolution code development) (LLNL)

- Experimental Validation (PNNL, LLNL)

At the end of FY2002, we have essentially completed our milestone for the void growth simulations implementing an earlier model for network dislocation evolution. Regarding our loop simulation milestone, we have analyzed experimental data and performed order of 
magnitude estimates for loop evolution. We find that loop mobility and coalescence dominate the late-time evolution. This necessitates a change of algorithm for evolving the loop size distribution functions. We believe the required change is well in hand. Preliminary loop evolution simulations should be achieved near the end of the first 12-15 project months, essentially on schedule for our first year milestones. Additionally over FY2002, we have constructed separate simulations of helium bubble evolution, in preparation for incorporating the formalism in an overall void/bubble/loop/dislocation microstructure code. We have further analyzed our irradiated samples, strengthening our interpretation of a dose-rate effect on incubation.

Below, we provide a more detailed description of our progress with the benefit of our current perspective. Further documentation is included in the form of our project publications for the year, appended to this report.

\section{Void nucleation and growth simulations. (Milestone 1, Tasks A and D)}

- We report software development success. The void-evolution computer code is fast, flexible, stable, and adequately conserves mass in all situations.

We have completed a microstructure code that evaluates the full, time-dependent distribution of vacancy clusters along with the dislocation density, all inside a mean-field framework. The vacancy cluster distribution is treated in full generality, ranging from monomers to voids of arbitrary size.

The stochastic, atomic processes of void growth or shrinkage are included by master equation and Fokker-Planck treatments of the void size distribution function. Void growth and fluctuation rates are determined from a self-consistent calculation of the thermal and radiation-induced vacancy and interstitial monomer populations. Currently, interstitial aggregation is not allowed, as dislocation loops are not explicitly considered. However, it will be included in the future. The dislocation subsystem is modeled entirely as network dislocations, in terms of a single density parameter. We have verified that our implementation of the dislocation evolution model reproduces earlier results for annealing in 316 stainless steel.

We devoted considerable effort to improving the conservation of mass sum rule. This entailed correcting for deficiencies in our Monte Carlo Langevin simulation and improving the selfconsistent solution of our monomer rate equations. We have repeated our early calculations using the new, improved code. Our new calculations are numerically reliable from 250 to $750 \mathrm{C}$, a vital requirement for application to Generation IV reactor designs. The computer code has been optimized in order to reach 150 dpa fluence with reduced computational effort.

- We report a major scientific success with our new method.

Our new simulations are the first ever to show a realistic incubation-like feature, reported at the Tucson ASTM STP 1447 conference. We also find a moderately lengthy period of steady-state swelling near the experimental rate of $1 \% / \mathrm{dpa}$ in austenitic stainless steels. We see that prolongation of the steady behavior requires the co-evolution of voids and dislocations. This is a major achievement from our first milestone - namely our simulated co-evolution of voids and network dislocations is in qualitative agreement with experiment.

We have applied our method to a model of a high-purity type- 316 austenitic stainless steel. 
It is easy to see a temperature-dependent incubation-like period in the simulated void swelling versus time. Volumetric swelling curves are presented in Fig. 1 for a series of temperatures $\left(340 \mathrm{C}\right.$ to $540 \mathrm{C}$ ) for an irradiation dose rate of $10^{-6} \mathrm{dpa} / \mathrm{s}$ and an initial dislocation density of $6 \times 10^{13} \mathrm{~m}^{-2}$, using a constant dislocation bias factor of 1.63 for interstitials and 1.04 for vacancies. Simulations show a brief incubation period. Subsequent swelling rates at different temperatures are comparable, around $0.85 \% / \mathrm{dpa}$. This rate is largely dictated by the dislocation bias factors. Overall, the predicted behavior is similar to experiment, both in the incubation-like feature and in the numerical value of the asymptotic slope, near the experimental value of $1 \% / \mathrm{dpa}$. The incubation period is short versus commercial steels, similar to pure binary and ternary austenitic steels.

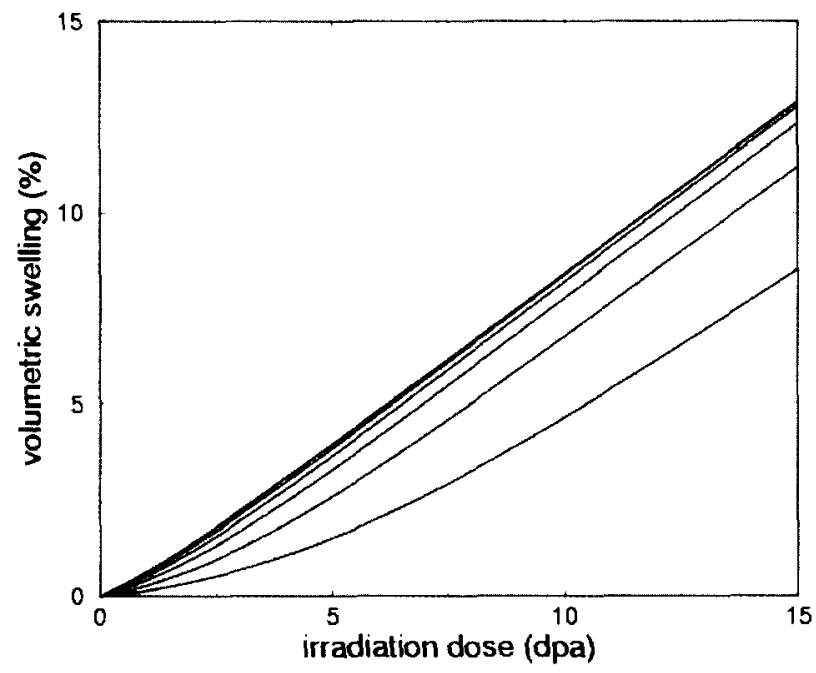

Fig.1 Volumetric swelling, $\Delta V / V$, versus total irradiation dose for pure, type 316 stainless steel. The dose rate is $10^{-6} \mathrm{dpa} / \mathrm{s}$; the starting dislocation density is $6 \times 10^{13} \mathrm{~m}^{-2}$. The various curves correspond to temperatures of $340 \mathrm{C}$ to $540 \mathrm{C}$ in increments of $40 \mathrm{C}$. The $340 \mathrm{C}$ curve has the longest incubation period. These calculations use constant dislocation bias factors.

- We discover a sensitivity of void evolution to dislocation bias factors. Dislocationdislocation correlation will influence the resulting bias factors and so influence void evolution.

We find that changing our definition for dislocation bias changes the void evolution, indicating that evolving dislocation-dislocation correlation functions will influence system behavior. Dislocation bias factors are calculated according to previously described methods (Sniegowski and Wolfer 1983). There is a cutoff distance used in the derivation that is related to the average density of dislocations. We make two choices for that parameter here. The first is to calculate dislocation bias factors assuming a density of $6 \times 10^{14} \mathrm{~m}^{-2}$, a typical terminal density under steady irradiation. This procedure would be expected give the experimentally observed asymptotic swelling rates for calculations with a fixed dislocation density. Our second choice is to calculate the bias factors using a cutoff obtained from the instantaneous value of the evolving dislocation density. That density is evolved according to a model (Wolfer and Glasgow 1985) that incorporates dislocation-dislocation annihilation processes along with a dislocation multiplication from pinned dislocations undergoing irradiation-driven, biased climb. The model includes one free parameter, namely, the mesh length or pinning density (set to a value of $400 \mathrm{~nm}$ to fit the observed terminal dislocation densities in irradiated steels), which is determined by the density of carbide precipitates in the steel. 
Our swelling predictions are very sensitive to the choice of dislocation bias factors. Fig. 2 displays the swelling curves using dislocation bias factors which are calculated according to the instantaneous dislocation density. The predicted dislocation bias factors are found to be smaller at low dislocation densities, for example 1.39 and (1.03) for interstitials (vacancies) at a dislocation density of $6 \times 10^{13} \mathrm{~m}^{-2}$, and increase with dislocation density. This gives rise to a prolonged period of void nucleation in solution-annealed metals, producing a striking, incubation-like behavior that lasts from 2 to $15 \mathrm{dpa}$ of total fluence. Besides lengthening the incubation period, the density-dependent bias also causes the asymptotic rates of swelling to depend noticeably on temperature. This change in behavior occurs because the terminal dislocation density is temperature-dependent, the calculated dislocation bias factors are now density-dependent, and the final swelling rates are strongly bias-dependent.

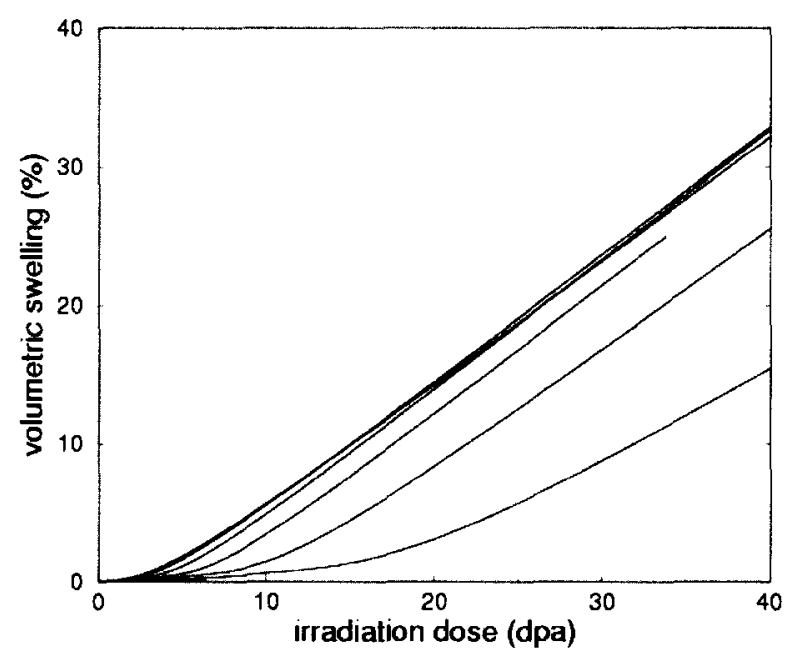

Fig. 2. Volumetric swelling, $\Delta V / V$ in percent, versus total irradiation dose (equivalently, the irradiation time, in Msec) for a pure, type-316 stainless steel. The system parameters are exactly as in Fig. 1, except that, here, the dislocation bias factors are allowed to vary with the dislocation density. Again, the lowest temperature curves display the longest incubation delays.

There is a striking difference in the predicted number density of voids between the constant and the variable dislocation-bias simulations, The predicted terminal void population is several times smaller with the variable dislocation bias than in the constant dislocation-bias case (Fig. 3). Essentially, small dislocation-interstitial bias factors at initial stages leaves a higher density of interstitials available to annihilate with vacancies. This reduces the vacancy supersaturation, delays void nucleation, and prolongs incubation. 


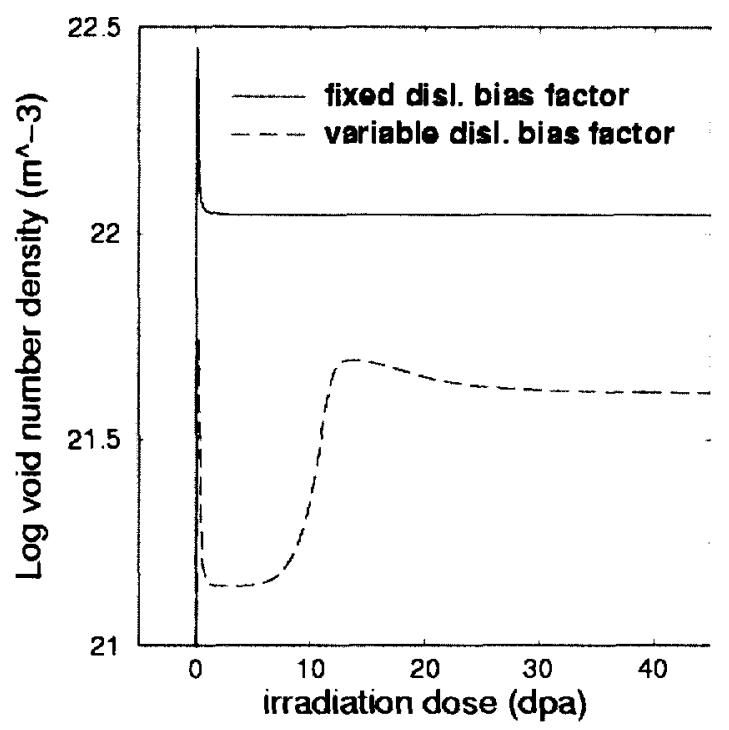

Fig. 3. "Visible" void densities (all voids of radius greater than $0.5 \mathrm{~nm}$ ) versus time in our void/dislocation co-evolution simulations. Both curves refer to calculations at $340 \mathrm{C}$ and dose rates of $10^{-6} \mathrm{dpa} / \mathrm{s}$. They differ only in the choice of dislocation bias factors. The top curve uses a constant dislocation bias factor of 1.04 (1.63) for vacancies (interstitials). The lower curve uses a density-dependent bias factor which starts at 1.03(1.39) for vacancies (interstitials), but evolves to the same values as the constant simulation.

- We see specific instances in which impurity effects are important. The mechanism must be identified and included our final model.

Our impurity-free model reproduces the short-incubation behavior of high purity metals (Fig. 4). However, commercial austenitic steels commonly possess incubation times of tens of dpa, compared to the delays of less than $5 \mathrm{dpa}$ in high-purity ternary alloys. The dominant mechanism for this effect has not been identified from among the candidate explanations.

- We obtain a dose-rate effect. This is the premiere achievement of our first year efforts.

Of particular importance, we see the "dose-rate" effect on incubation, namely, that the lower dose rate requires as smaller cumulative fluence to swell by $1 \%$. This is shown in Fig. 4. For moderate temperatures, the lowest dose rates reach $1 \%$ swelling at the lowest cumulative dose. 


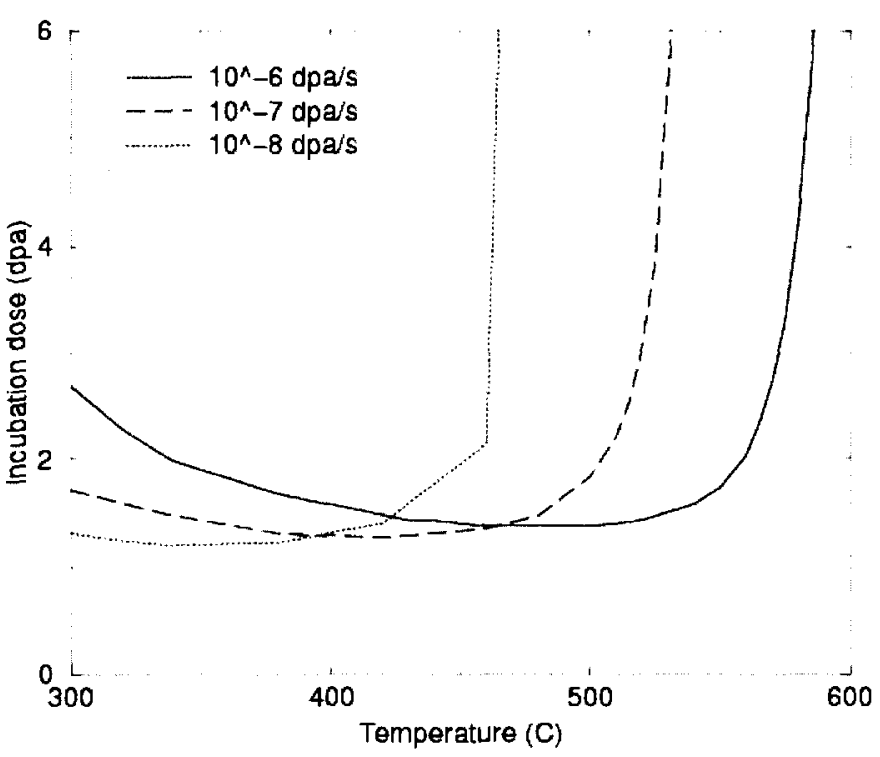

Fig. 4. Incubation dose versus temperature and three dose rates; $10^{-6}$ (solid line), $10^{-7}$ (dashed line), $10^{-8} \mathrm{dpa} / \mathrm{s}$ (dotted line) for a simulation with a constant dislocation density of $610^{14} \mathrm{~m}^{-2}$. We obtain short incubation times (typically 1-5 dpa), in agreement with data for high-purity, ternary stainless steels.

In summary, the present microstructural code for void nucleation and concurrent network dislocation evolution is generating swelling-fluence-temperature correlations which closely resemble what is obtained by Garner from recent data sets on EBR-II irradiated 304 stainless steels. At the same time, the new correlations are dramatically different than the empirical swelling design equations still in use for LWR and breeder reactors. In particular, we find that irradiation temperature does not have a large effect on the steady-state swelling rate, but rather a large influence on the incubation dose.

\section{Loop nucleation and growth simulations. (Milestone 2, Task B)}

- We have modified the nucleation and growth code in preparation for incorporating loop growth from monomer aggregation. We will be able to solve for the growth from pointdefect monomers.

We have restructured the basic master and Fokker-Planck microstructure evolution code so that both dislocation loops and helium bubbles may be added to the simulation. We will approximate prismatic dislocation loops as ideally circular. Bias factors and monomer absorption cross sections for such loops are already available from the literature. Suitable approximations for loop energy versus loop size are also available. Accordingly, the algorithm for loop growth from mobile monomer aggregation will be straightforward to include.

In order to treat loop growth in the presence of a concentration of vacancies, it is necessary to know the vacancy chemical potential at which the loop will neither grow nor shrink on average. This is obtained from the vacancy chemical potential, $\mu_{\text {vac }}$, and the loop energy, $\mathcal{E}(N)$, versus size, as $\frac{d \mathcal{E}}{d N}=\mu_{\text {vac }}$.

The radius of a circular loop (related to reciprocal curvature) is easily obtained by: 


$$
N \Omega=\pi R^{2} b
$$

in terms of burgers vector, $b$, atomic volume, $\Omega$, and radius, $R$. Thus, transforming to functions of radius:

$$
\frac{d \mathcal{E}}{d N}=\frac{\Omega}{2 \pi b R} \frac{d \mathcal{E}}{d R}
$$

The energy of a circular loop versus radius is given by F. Kroupa (Czech. J. Phys. 10, 284 (1960))

$$
\mathcal{E}=\frac{G b^{2} R}{1-v} \frac{b}{R}\left\{K\left(1-\frac{r_{0}}{R}\right)-E\left(1-\frac{r_{0}}{R}\right)\right\} \cong \frac{G b^{2} R}{2(1-v)}\left\{\log \left(\frac{8 R}{r_{0}}\right)-1\right\}
$$

in terms of elliptic integrals $E$ and $K$, which is approximately equal to the right hand side when $R>>r_{0}$, the dislocation core radius.

Replacing the core radius, $r_{0}=b$, and using dimensionless $R / b$ :

$$
\Delta \mu=\mp \frac{G \Omega}{2 \pi(1-v)} \frac{b}{R} \frac{d}{d(R / b)}\left\{\frac{R}{b}\left[K\left(1-\frac{b}{R}\right)-E\left(1-\frac{b}{R}\right)\right]\right\}
$$

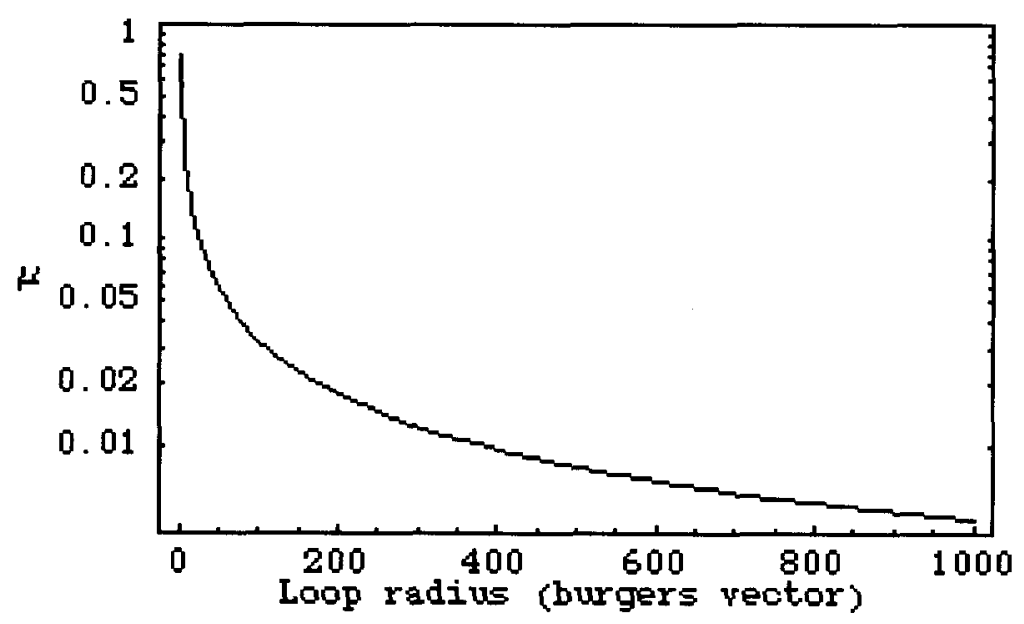

Fig. 5. Bulk vacancy chemical potential for a prismatic vacancy loop versus loop radius. The excess chemical potential approaches zero as the loop becomes straight. There is an analogous function for the equilibrium vacancy concentration for voids used in the void evolution code.

This allows us to model loop growth/annihilation from the vacancy monomers in solution using exactly the same computational procedure as for voids.

- We have identified an important coalescence mechanism for late-stage loop evolution. We have simple test-case solutions for interstitial loop coalescence by pipe diffusion.

Dr. Okita formally joined our project in August 2002, after completion of his Ph.D. thesis. As a result, much of our work on loop evolution began towards the end of FY 2002. The examination 
of Dr. Okita's experimental data and the concurrent examination of order-of-magnitude theoretical estimates demonstrate that loop glide plus climb mobility control loop evolution.

We treat the case of two prismatic loop on parallel planes, separated from each other as shown in the sketch. Foreman and Eshelby give as the mutual climb force for distances $x>2 D$ the result

$$
\begin{aligned}
& F_{c}=\frac{\mu b^{2}}{4(1-v)} \frac{\pi r^{4} \sin ^{4} \theta}{x^{4}} g(\theta) \\
& g(\theta)=3 \sin \theta\left(1+10 \cos ^{2} \theta-35 \cos ^{4} \theta\right)
\end{aligned}
$$

If we assume that pipe diffusion is the dominant mechanism for loop migration by climb, the velocity of approach of the two centers is given by

$$
v=\frac{d x}{d t}=-\frac{12 \pi}{1-v} \frac{\mu \Omega}{k T} D_{p} \frac{r b^{2}}{x^{4}} \sin ^{4} \theta g(\theta)
$$

Consider the case of coplanar loops $(\theta=0)$. Then

$$
\frac{d x}{d t}=-K(T) x^{-4}
$$

Integration from $x=2 D$ to $x=2 r$ gives the time required for the loops to touch. Hence.

$$
\begin{aligned}
& K(T) t_{\text {coalesce }}=\frac{32}{5}\left(D^{5}-r^{5}\right) \\
& t_{\text {coalesce }}=\frac{8(1-v)}{45 \pi} \frac{k T}{\mu \Omega} \frac{r^{4}}{b^{2} D_{p}}\left[(D / r)^{5}-1\right]
\end{aligned}
$$

or

Use $b^{2} / D_{p}=6.5 \cdot 10^{-16} \exp (-1.5 \mathrm{eV} / k T) \sec$ onds.

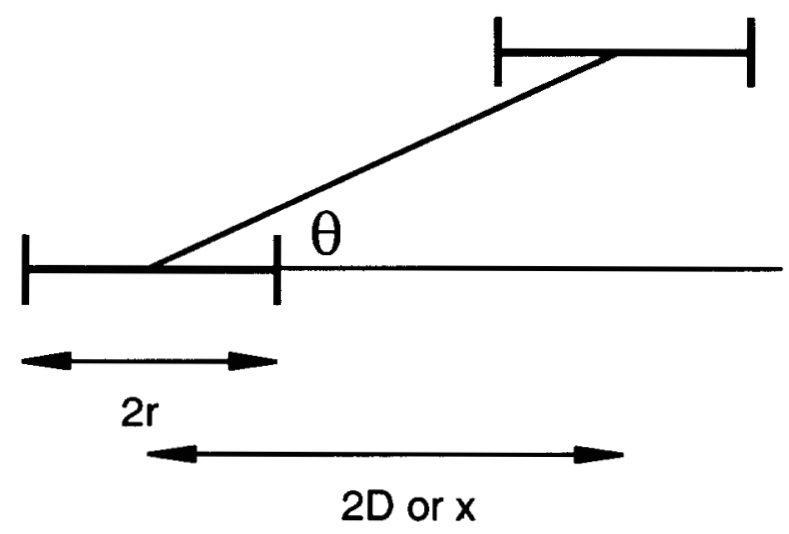

Fig. 6. Spatial relations of two prismatic dislocation loops on parallel planes used in the equations for loop climb motion. Here, $\theta$ should be the angle between the normal to the plane of the loops and the vector between the two loop centers. 
Given the motion of dislocation loops under stress, we expect to see loop-loop and loopdislocation impingement, and direct coalescence of these defect clusters. We have performed a search of the literature during FY02 and have already located a suitable algorithm for including cluster coalescence.

\section{Detailed void/dislocation co-evolution beyond the mean field. (Task C)}

- A simple parametrization for dislocation kinetics has been implemented. During this implementation we have identified difficulties with the original definition for atomic chemical potential along the curve.

Our initial problems have been eliminated, and work is underway to model the evolution of a dislocation that intersects a void. The effective, aggregate bias and the net mobile defect influx to this composite defect will be important to our models of late-time evolution under irradiation.

- The formalism for the detailed climb evolution of curved dislocations in a solution of bulk point-defect monomers has been established.

Our formalism begins with an approximation for the chemical potential of atoms along a dislocation segment with an arbitrary curvature in a climb plane. We replace a short, curved segment with a comparable portion of a circular prismatic dislocation loop of the same local curvature. The bulk vacancy concentration in equilibrium with the prismatic loop is

$$
C_{v}^{l o o p}=C_{\nu}^{e q} e^{[-\beta d E / d N]}=e^{\mid \beta \mu]}
$$

in terms of $\beta=1 / k_{B} \mathrm{~T}$ and the bulk vacancy concentration in equilibrium with a straight edge dislocation, $C_{v}{ }^{e q}$. The local atomic chemical potential of the curved dislocation is, therefore, given by the differences in loop energy, $\mathcal{E}$, on adding or subtracting matter, exactly as described for the circular loop problem in Milestone 2 and Task B.

\section{Bubble nucleation and growth simulations. (Tasks A and D.)}

Helium production and void nucleation are expected to under similar irradiation conditions. They may compete or synergize with each other, or simply occur in parallel. To investigate this process, we have developed a separate numerical simulation of He bubble development. We chose this route, rather than implement concurrent bubble/void simulations from the outset, for several reasons. The results provide perspective for comparison to experiment, benchmarks for the planned, integrated void/bubble evolution models, and encouragement that our projected approach to this problem will be both a sound and a fruitful one. Our study suggests that it is appropriate to consider formation of helium bubbles as inevitable, unless a sufficiently high void density exists already as traps before and during helium production. The roles of helium and void swelling can be reversed from conventional expectations; void swelling may prevent helium bubble formation rather than helium enabling and controlling void swelling.

The ratio of He production to dpa rate varies widely from fast neutron to thermal reactors, especially in Ni containing alloys like austenitic stainless steels, where a two-step neutron capture by ${ }^{58} \mathrm{Ni}$ ultimately results in alpha decay. (The accumulated helium content in pure nickel irradiated in HFIR is shown in Fig. 7.) In such alloys, the helium generation rate builds up gradually, delaying the formation of helium bubbles to higher doses. Depending on the He/dpa ratio, void swelling can intervene in bubble formation and even suppress it. The delay of $\mathrm{He}$ 
production in Ni-containing stainless steels to some 10 dpa allows voids to nucleate and grow. Void swelling results from EBR-II and HFIR irradiations have shown that voids may develop unaffected by helium generation, although abundant nanometer-sized helium bubbles are ultimately found in HFIR and none in EBR-II irradiated materials.

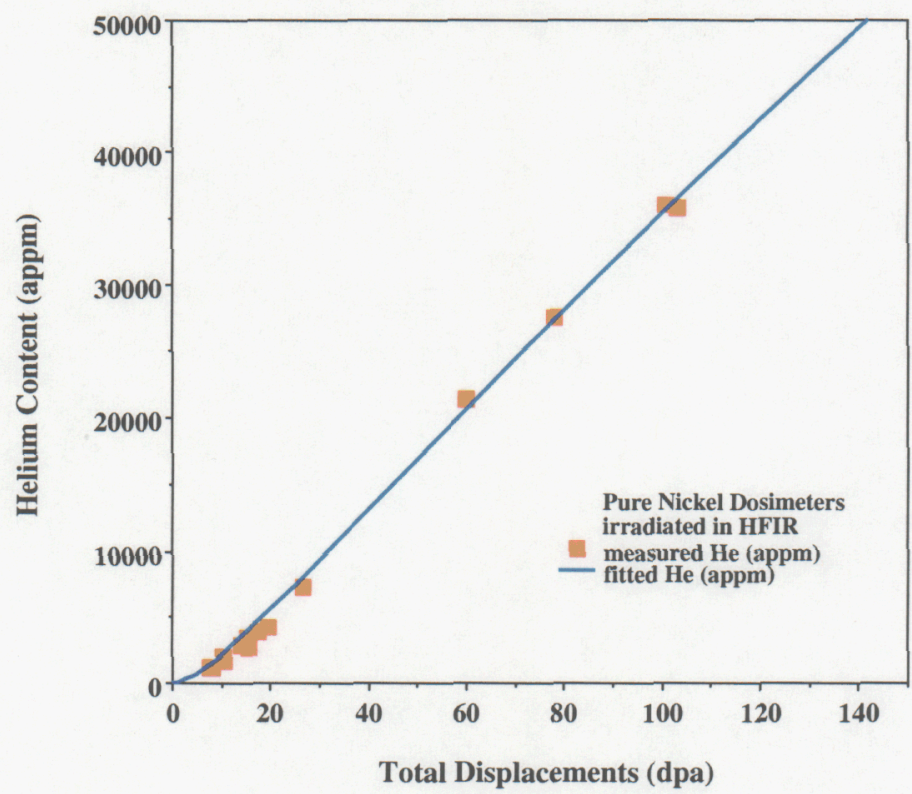

Figure 7. Helium produced in Nickel Dosimeters irradiated in HFIR. The fit is obtained by empirically adjusting parameters for the two-step nuclear reaction.

Our accomplishments for FY2002 include:

- We successfully simulate He bubble formation with and without pre-existing voids. We develop a database of helium bubble size distributions for different reactor situations for benchmarking integrated bubble/void calculations.

We consider kinetic models of increasing complexity for the formation and growth of bubbles. He bubble evolution is then obtained by solution of coupled rate equations. At temperatures and under irradiation conditions where self-diffusion is unimportant, helium bubble formation can be considered as an irreversible aggregation process. The dominant transport mechanism is the vacancy-coupled diffusion of helium monomers.

Helium dimers and trimers may also be mobile, and their growth by the capture of monomers may be enhanced by their own mobility. While this could easily be included in the analysis, we do not explicitly include this effect. Similarly, we neglect reactions of coalescence between the clusters.

Results of calculations using both a (simplified) constant He generation and the (realistic) measured dose-dependent generation function are shown below. Under constant helium generation rates, we find that the helium concentration in solution peaks sharply so that nucleation occurs very rapidly over a very short period of time. After this initial nucleation burst, the helium concentration in solution remains low as bubbles already present quickly capture newly generated helium. Bubbles of similar age grow at roughly similar rates and stay at similar sizes, so a narrow peak in the size distribution persists. For the constant generation rates of 200 appm/dpa and 400 appm/dpa (Fig. 8 and 9, respectively), we observe a sharp peak in the 
distributions at the largest sizes, corresponding to the early burst of nucleation. Higher generation rates result in a slightly larger number of bubbles $/ \mathrm{cm}^{3}$.

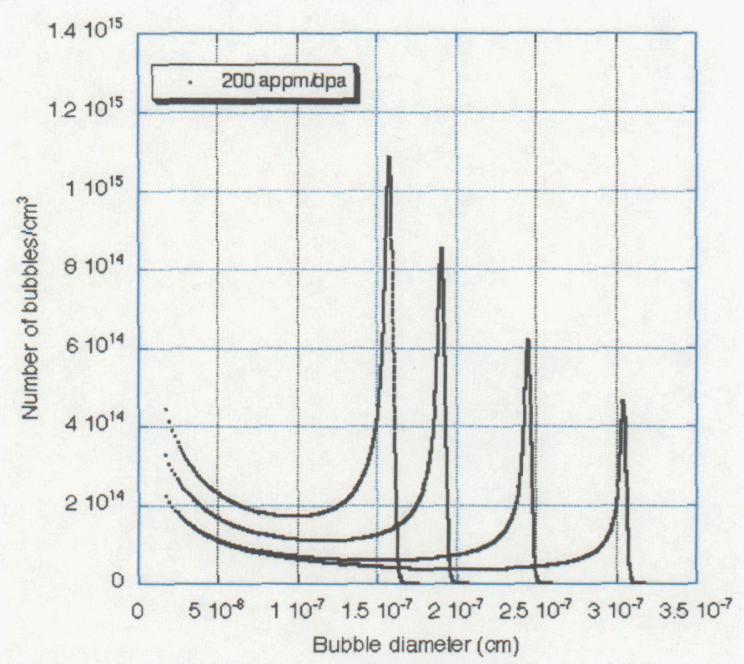

Figure 8. He bubble size distributions in $\mathrm{Ni}$ as the bubbles evolve over time at $350{ }^{\circ} \mathrm{C}$. The curves indicate the size distributions at 1 year, 2 years, 5 years and 10 years using constant $\mathrm{He}$ generation rates of $200 \mathrm{appm} / \mathrm{dpa}$. Many bubbles nucleate together in a sharp burst near the outset - these contribute to the narrow peak of bubbles of similar age and size. The peak height drops with successive plots because the size distribution is transformed to radial coordinates.

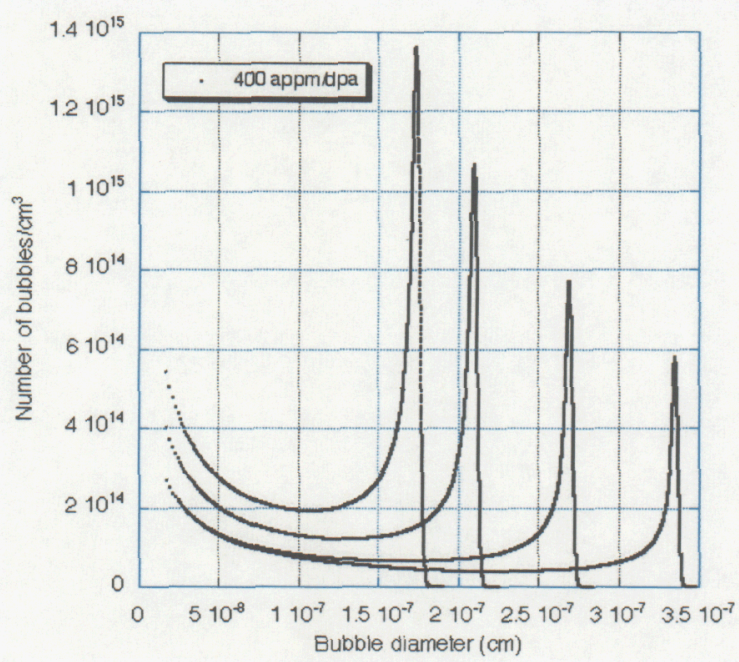

Figure 9. He bubble size distributions in $\mathrm{Ni}$ as the bubbles evolve over time at $350{ }^{\circ} \mathrm{C}$. The curves indicate the size distributions at 1 year, 2 years, 5 years and 10 years using constant He generation rates of $400 \mathrm{appm} / \mathrm{dpa}$.

Figure 10 shows the size distribution resulting from the dose dependent generation rate. The general features of the bubble size distributions are a leading peak and a tail that connects it to the embryonic clusters, in our case to the dimers. These results show that a gradual increase of the helium generation rate dramatically reduces the early nucleation burst. As a result, the density peak at largest sizes is much reduced. The presence of early bubbles and the slow increase in production rates causes the helium monomer density to change more gradually. Bubble nucleation persists for longer times, albeit at much lower rates. Thus, the initial peak of early clusters merges with the tail of smaller ones. 


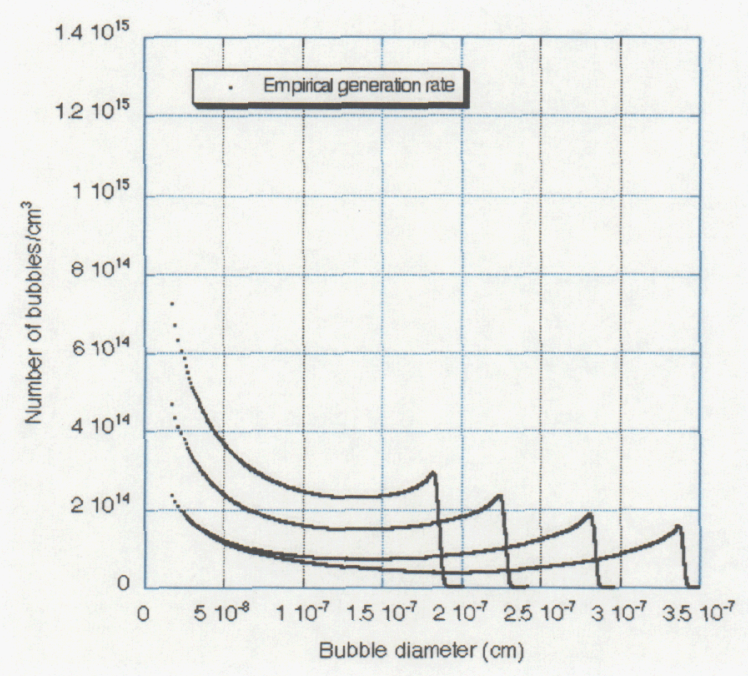

Figure 10. He bubble size distributions in $\mathrm{Ni}$ as the bubbles evolve over time at $350{ }^{\circ} \mathrm{C}$. The curves indicate the size distributions at 1 year, 2 years, 5 years and 10 years using the empirical fit to He production due to ${ }^{58} \mathrm{Ni}$ transmutation.

The resulting bubble size distribution is narrower than seen experimentally, even when the gradual onset of He production is accounted for. This is expected, since real materials have local inhomogeneities that influence bubble nucleation. Similarly, variations in alloy stoichiometry will affect local diffusivities and make the bubble formation nonuniform. Both effects will act to broaden the expected size distribution, but they have little effect on the total bubble density.

We also obtain bubble densities for the different helium generation models.

The burst of nucleation with a constant helium production is clearly visible in Fig. 11. Our results in Fig. 12 show that the gradual increase of helium generation dramatically reduces the early nucleation burst and results in a more uniform rate of nucleation.

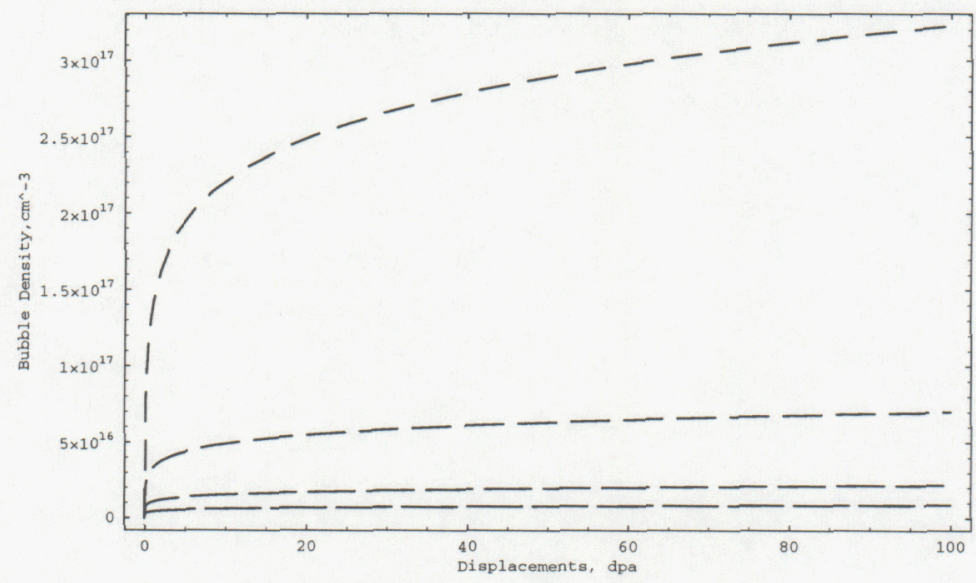

Figure 11. Helium bubble densities predicted for type 316 stainless steels in HFIR at 350, 450, 550 , and $650^{\circ} \mathrm{C}$. Higher temperature gives lower bubble densities. This shows the results for a hypothetical average generation rate of $50 \mathrm{appm} / \mathrm{dpa}$. 


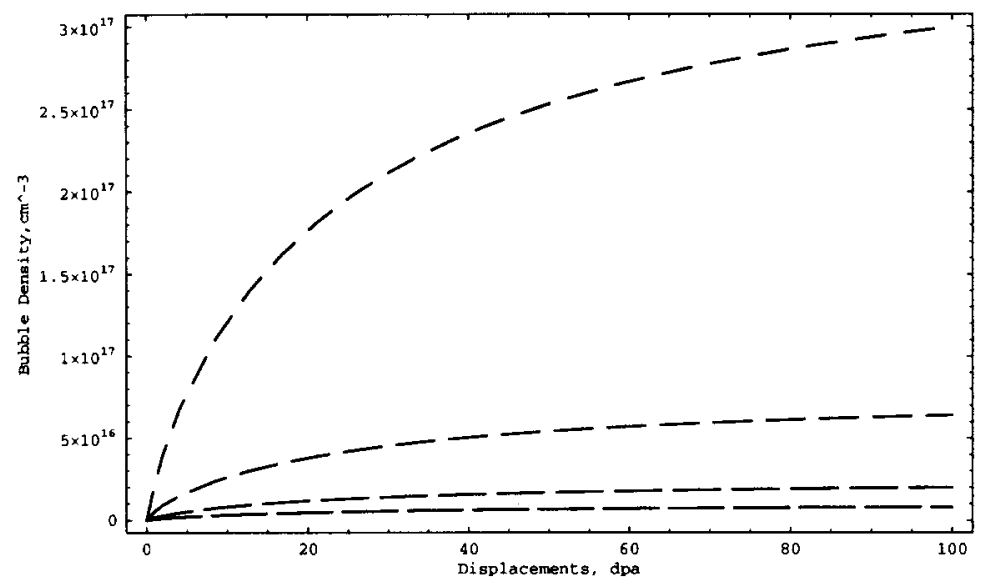

Figure 12. Helium bubble densities predicted for type 316 stainless steels irradiated in HFIR at temperatures of $350,450,550$, and $650^{\circ} \mathrm{C}$. The higher the temperature, the lower the bubble densities (and the longer the dashes). This is for the experimentally determined dose dependent helium generation.

- We discover that early void formation can depress bubble densities. Voids are effective sinks for helium in solution.

Finally, we solve for He bubble formation with a pre-defined density of voids to serve as $\mathrm{He}$ sinks. The time-dependent void densities are taken from our separate simulations. Once voids are present, the number of bubbles with a high density of He develops much more slowly. Also the asymptotic number density is reduced by an order of magnitude.

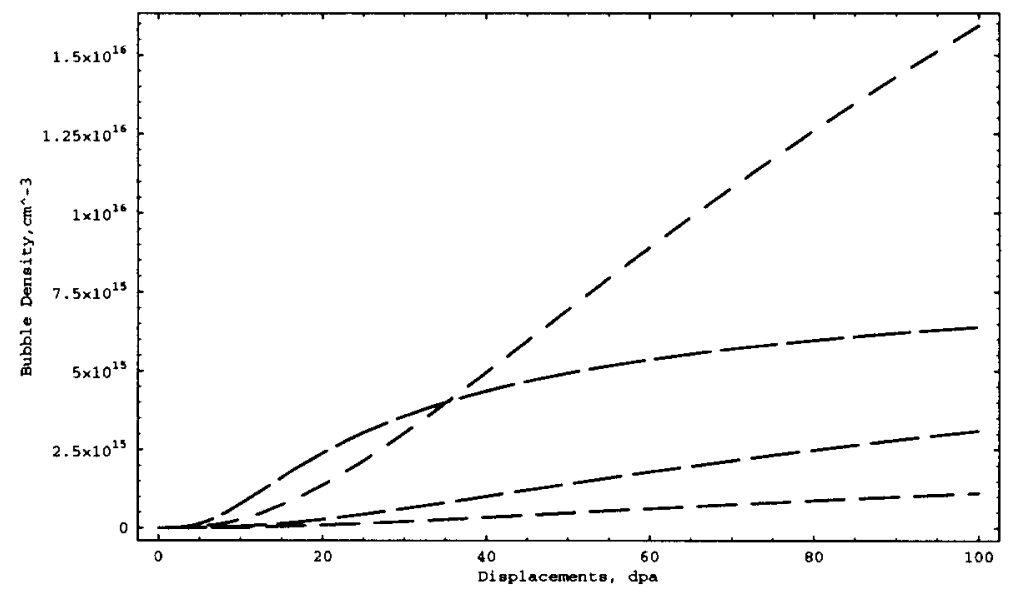

Figure 13. Helium bubble formation in the presence of voids when they evolve according to results from our (He-free) void nucleation and growth simulations. Void densities range from 2 $10^{16}$ to $110^{15}$ depending on temperature. Increasing void formation suppresses the bubble nucleation rate.

- We derive universal behaviors to provide benchmarks for the projected combination void/bubble code. The evolution of bubble density and average radius approximately follows a universal curve when dimensionless variables are introduced. 
We identify scaling relationships involved in mapping experimental bubble densities onto the universal curve that provide the means to extract a helium diffusion coefficient. With suitable approximations for the bubble distribution, and when the helium generation rate is a constant, it is possible to introduce dimensionless quantities and transform the three differential equations to a set which no longer contains the generation rate and the diffusion constant. These three differential equations have, for the initial conditions of vanishing monomer and cluster concentrations, one unique solution, which we shall call the master solution. All real solutions to the rate equations with vanishing initial conditions but different temperatures or production rates can be constructed from this master solution. The master curve for the bubble density is shown in Figure 14.

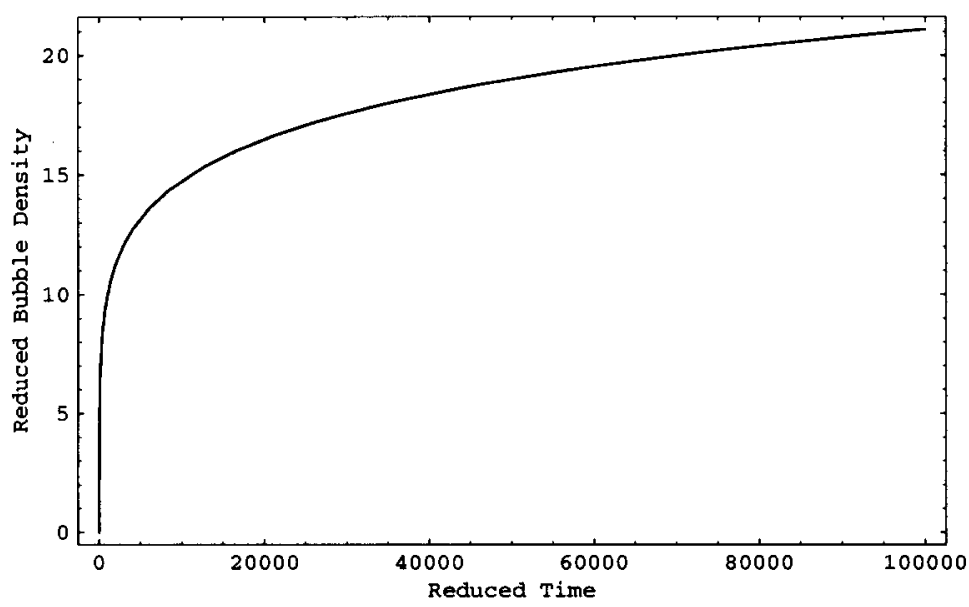

Figure 14. The master curve for bubble density evolution for constant helium generation rate, $G_{0}$.

The scaling parameter which connects all real solutions to the master solution is given by

$$
A\left(D, G_{0}\right)=\frac{1}{\Omega} \sqrt{\frac{4 \pi r_{1} D}{G_{0}}}
$$

in terms of diffusivity, D, monomer radius, $r_{1}$, atomic volume $\Omega$. The bubble density is

$$
n(\tau)=A \Omega N_{B}(t)
$$

in reduced, dimensionless form, and where the reduced time is

$$
\tau=A \Omega G_{0} t
$$

To lend meaning to these reduced quantities, let us suppose that helium bubble density measurements have been made by TEM on samples of the same steel exposed in a nuclear reactor at various locations throughout the core. Each sample has associated with it an irradiation temperature, $T_{i}$, an irradiation exposure, $G_{o i} t_{i}$ and a bubble density, $N_{B i}$. If one knows the diffusion coefficient, a particular value, $D_{\mathrm{i}}$, can be assigned to each sample, and the scaling parameter, $A_{\mathrm{i}}$, the reduced bubble density, $n_{\mathrm{i}}$, and time, $\tau_{\mathrm{i}}$, can be computed. A plot of $n_{\mathrm{i}}$ versus $\tau_{i}$ should place the data on the master curve. Systematic deviations from the master curve may imply an inaccurate diffusion coefficient, and by adjusting its parameters to obtain the least 
deviation, it can in fact become a method to determine the diffusion coefficient for helium during irradiation.

The bubble evolution for a constant helium generation rate exhibits a very rapid rise. To appreciate this phenomenon, let us look at the monomer concentration, i. e. at the helium in solution as a function of time. In reduced units, the helium in solution (Fig. 15) is given by

$$
c(\tau)=A \Omega N_{1}(t)
$$

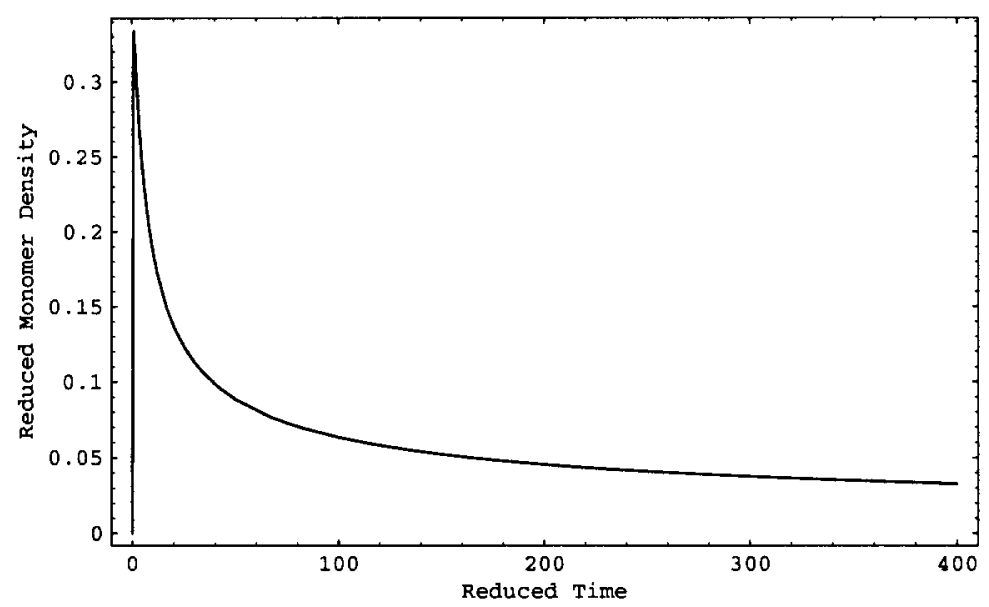

Figure 15. The master curve for the reduced helium concentration in solution. Note, that only the initial part is shown compared to the results shown in Figure 12.

- Our computer code is available to incorporate in the overall microstructure evolution code.

We have written the overall microstructure code to easily incorporate helium bubbles. However, if desired, we can add the existing bubble evolution code as a separate component in the system evolution.

\section{Experimental Validation (Task E)}

We have surveyed the results of numerous recent investigations conducted on austenitic steels removed from either fast reactors or thermal power reactors of both Western or Russian design. These experiments provide some insight on the potential for void swelling in PWRs at lifetimes in the 40-60 operating year range. The implications are listed here and in the attached publication by Garner, et. al.

- Void swelling is definitely observed under conditions typical of PWR.

There have been several observations of void swelling in Western PWRs and VVERs of Russian design. Edwards and coworkers have recently published the results of an examination of a baffle-former bolt removed after 20 years from the Tihange 1 reactor. This bolt, designated $2 \mathrm{~K} 1 \mathrm{R} 5$, was constructed from $15 \%$ cold-worked 316 stainless steel, and was exposed at former level 5 to a maximum of $19.5 \mathrm{dpa}$ at its head, and $\sim 7 \mathrm{dpa}$ at the top of the threads, as determined by retrospective dosimetry by Garner and coworkers. The bolt was sectioned to observe its microstructure at positions 1,25 and $55 \mathrm{~mm}$ from the surface of the bolt head. The calculated temperatures at mid-cycle conditions for these sections were 320,345 and $330^{\circ} \mathrm{C}$, respectively, as shown in Figure 16. 


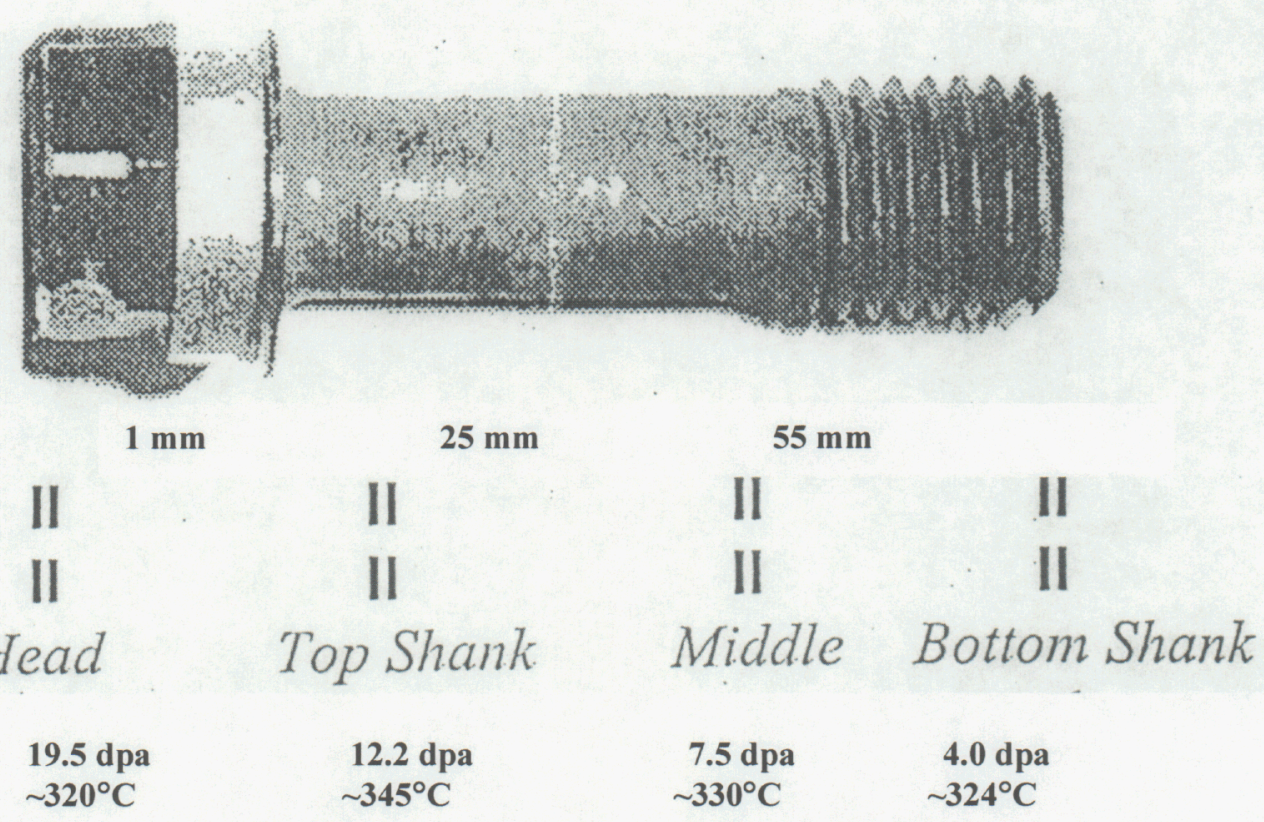

Figure 16. Irradiation conditions experienced by the 2K1R5 baffle-former bolt at the positions chosen for microstructural examination.

Cavities in the form of bubbles or voids were observed in the matrix of all three sections, but the swelling at $1 \mathrm{~mm}$ was very low $(<0.01 \%)$ with clusters of small voids/bubbles distributed very heterogeneously. Larger voids were much better defined and homogeneously distributed at the 25 and $55 \mathrm{~mm}$ positions, with swelling at 0.20 and $0.24 \%$, respectively. Note the clearly faceted sides of the voids, as shown in Figure 17.

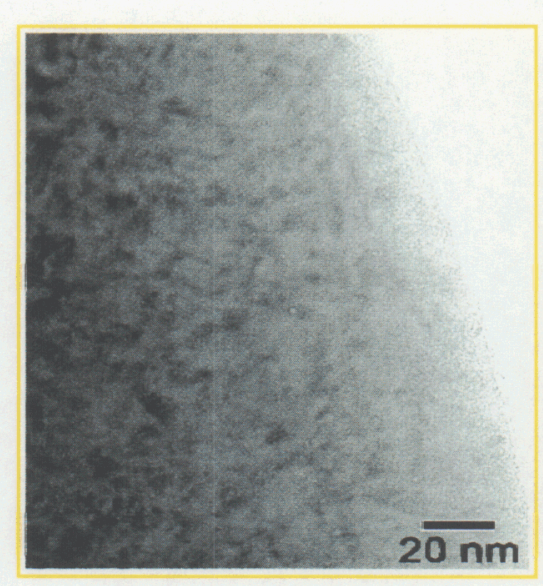

Bolt Head, $0 \mathrm{~mm}$ $19.5 \mathrm{dp}, \sim 320^{\circ} \mathrm{C}$

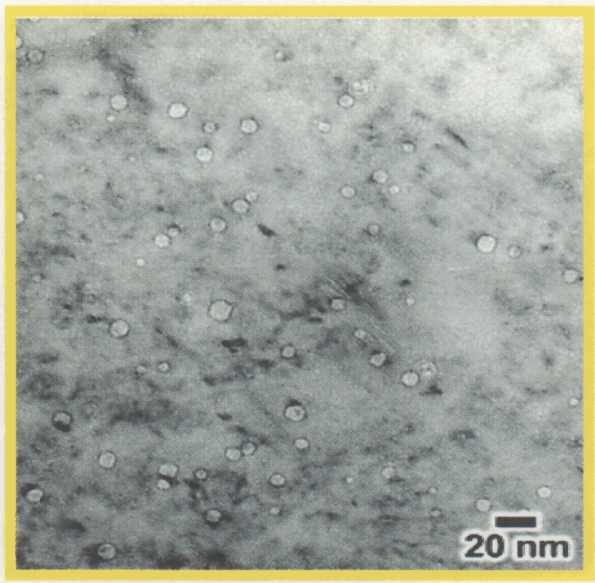

Top Shank, $25 \mathrm{~mm}$ $12.2 \mathrm{dp}, \sim 340^{\circ} \mathrm{C}$

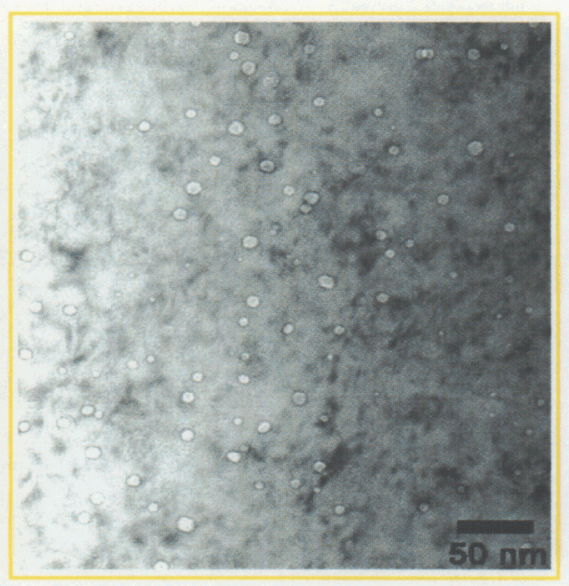

Near Threads, 55 mm $7.5 \mathrm{dp},-330^{\circ} \mathrm{C}$

Figure 17. Voids observed in the 2K1R5 bolt sections examined by electron microscopy.

- Void swelling never seems to occur below $300 \mathrm{C}$.

In the current experiment $12 \mathrm{X} 18 \mathrm{H} 10 \mathrm{~T}$, the Soviet analog of AISI 321 , was used as a flow restrictor component in the reflector region of the BN-350 fast reactor located in Kazakhstan 
(Porollo et. al.). This component experienced conditions that spanned the range of 4 to 56 dpa at $1.1 \times 10^{-8}$ to $1.6 \times 10^{-7} \mathrm{dpa} / \mathrm{sec}$ and $270-340^{\circ} \mathrm{C}$, after residing in the reactor for 12 years. With the exception of the neutron spectral differences, primarily in the lower generation rate of transmutant helium and hydrogen, these fast reactor conditions are very similar to the conditions experienced by PWR internals.

The approach used in this experiment was to cut the central $1.5 \mathrm{~m}$ section of this component and then section it into many small microscopy disks, each with a relatively well-defined dpa, dpa rate and irradiation temperature. The microstructures of 157 specimens were examined, and voids were found in 111 of these. Also observed in all specimens were irradiation-produced Gphase precipitates, dislocation loops and lines.

Figure 18 shows the swelling observed for all specimens, irrespective of dpa and dpa rate, that were irradiated above $300^{\circ} \mathrm{C}$, with swelling accelerating strongly above $\sim 310^{\circ} \mathrm{C}$. There were no voids observed below $300^{\circ} \mathrm{C}$. The maximum swelling was only $\sim 1 \%$ at $\sim 50 \mathrm{dpa}$ and $\sim 330^{\circ} \mathrm{C}$. There is a very strong effect of increasing irradiation temperature to accelerate swelling even as the dpa level falls. Analysis of the swelling data show that in this limited range of temperatures the effect of falling dpa rate is to accelerate the onset and level of swelling.

There appears to be a lower temperature boundary of $\sim 300-305^{\circ} \mathrm{C}$ below which voids were not observed. These data agree very well with the VVER and BWR observations of Neustroev.

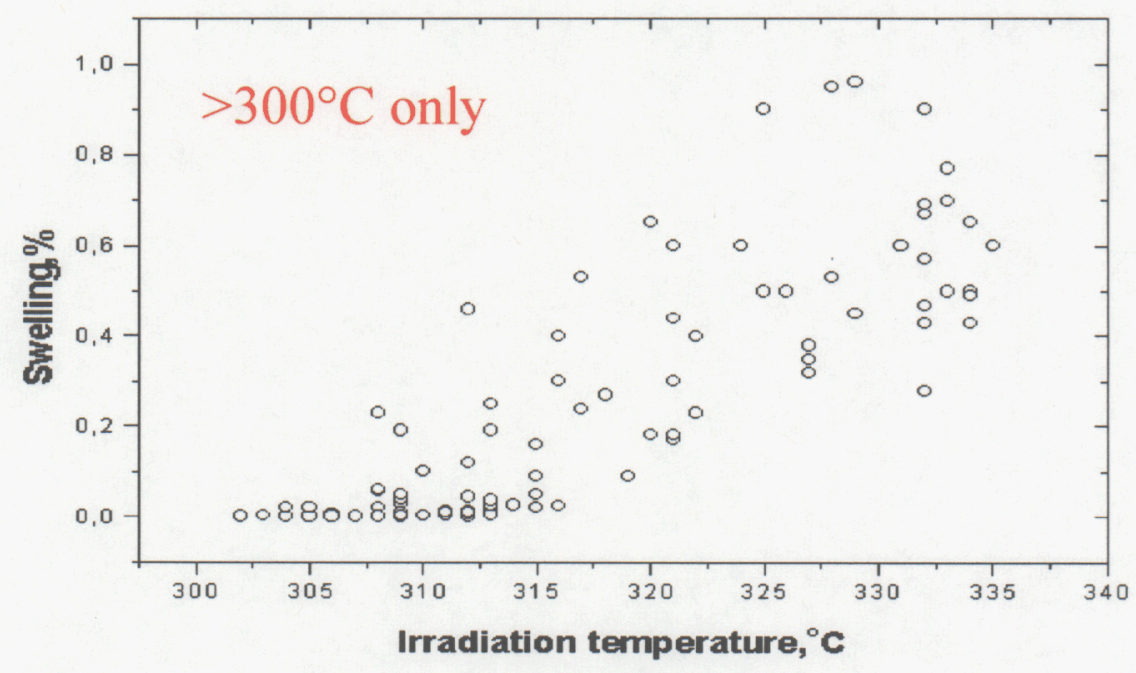

Figure 18. Swelling levels vs. irradiation temperature measured by microscopy in the annealed $12 \mathrm{X} 18 \mathrm{H} 10 \mathrm{~T}$ flow restrictor irradiated in the low-flux reflector region of $\mathrm{BN}-350$ fast reactor. The dpa levels vary from about 4-56 dpa. Irradiation temperatures ranged from 270 to $340^{\circ} \mathrm{C}$, but no voids were observed below $\sim 300 \mathrm{C}$.

- The incubation dose rate effect is generally observed.

Much of the data we analyze imply that swelling increases at lower dpa rates, possibly by reducing the duration of the transient regime of swelling. In order to conclusively demonstrate the effect of dpa rate on void swelling Garner and coworkers conducted a much more 
comprehensive experiment on annealed AISI 304 stainless steel. This experiment isolated the effect of dpa rate by concentrating on a limited range of temperatures $\left(373-444^{\circ} \mathrm{C}\right.$, with the majority of the data at $\left.373-410^{\circ} \mathrm{C}\right)$, but a very large range of dpa rates $\left(0.06-3.8 \times 10^{-7}\right.$ $\mathrm{dpa} / \mathrm{sec}$ ), with no significant difference in helium/dpa ratio. These temperatures are characteristic only of the upper range of PWR interest, and not the lower range experienced by the majority of the internals, but the dpa rates span the full range of PWR internal conditions.

The experiment involved the examination of five unfueled hexagonal subassemblies constructed of a single heat of annealed AISI 304 stainless steel irradiated for many years in the reflector rows $8,9,10$ and blanket row 14 of the EBR-II fast reactor. A total of 280 disks $(2 \mathrm{~cm}$ diameter by $1 \mathrm{~mm}$ thick) were cut from these assemblies. Each disk had a unique combination of average irradiation temperature, dpa and dpa rate, produced by both axial and radial variations in these parameters. Swelling was measured by immersion density for all 280 disks and was confirmed by microscopy on 40 specimens spanning the full range of the experiment. Voids were found in all examined specimens with swelling ranging as high as $2.8 \%$. Examples of the swelling and its sensitivity to dpa rate are shown in Figure 19.

(a)
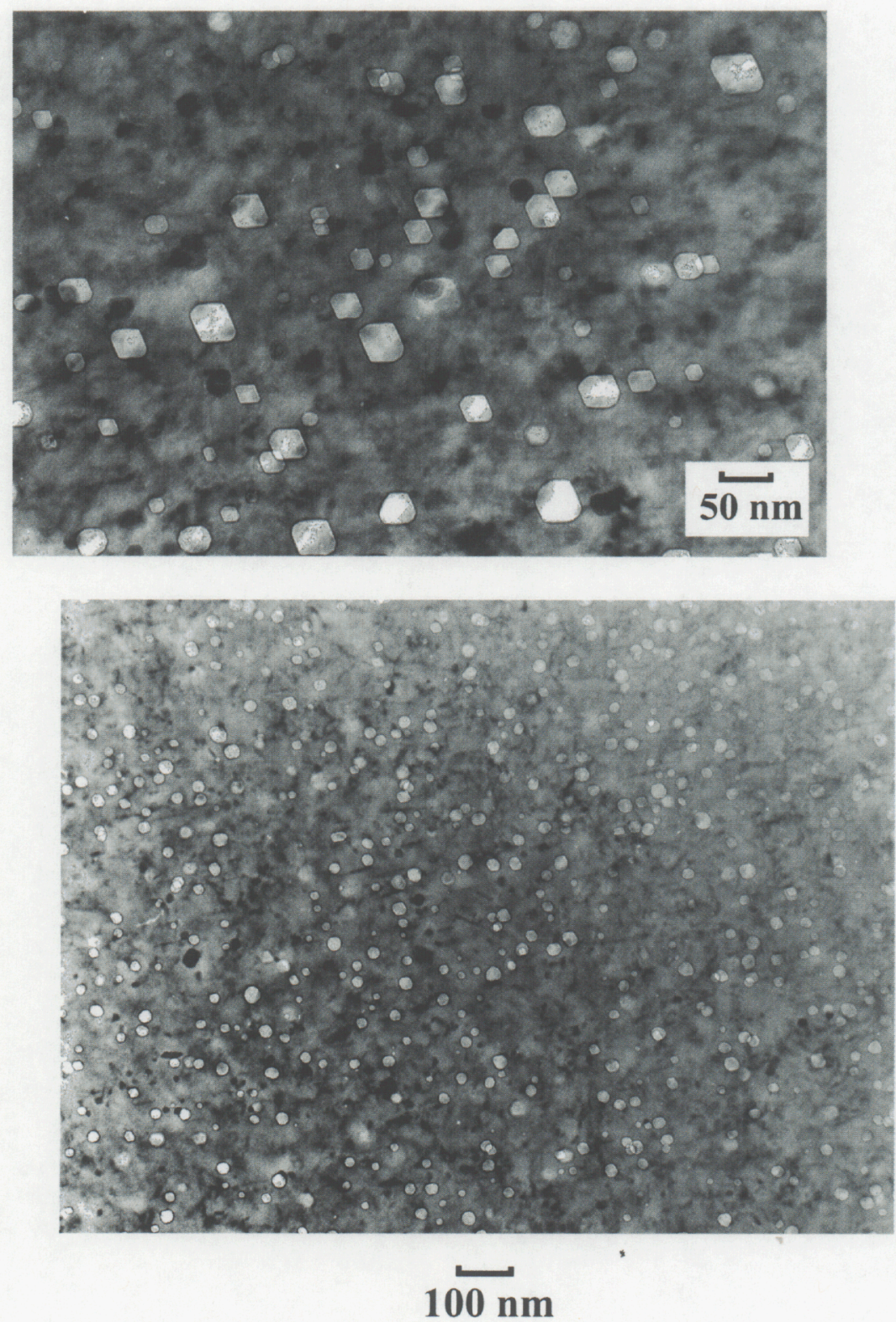
Figure 19. Demonstration of the effect of dpa rate on swelling of annealed AISI 304 stainless steel in EBR-II. a) Void microstructure at $380^{\circ} \mathrm{C}$ and $21.7 \mathrm{dpa}$ at $0.84 \times 10^{-7} \mathrm{dpa} / \mathrm{sec}$, reaching $1.0 \%$ swelling. b) Void microstructure at $378^{\circ} \mathrm{C}$ and only $10 \mathrm{dpa}$ at $0.15 \times 10^{-7} \mathrm{dpa} / \mathrm{sec}$, reaching $1.2 \%$ swelling. Note the change of scale between the two micrographs.

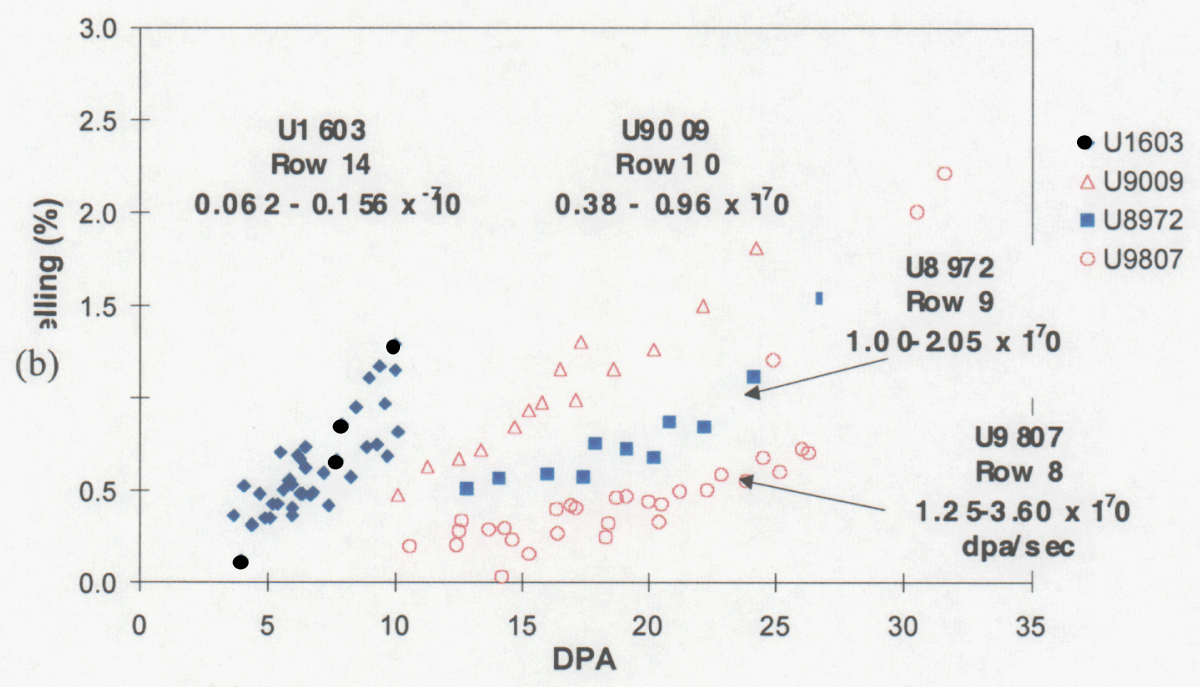

(a)

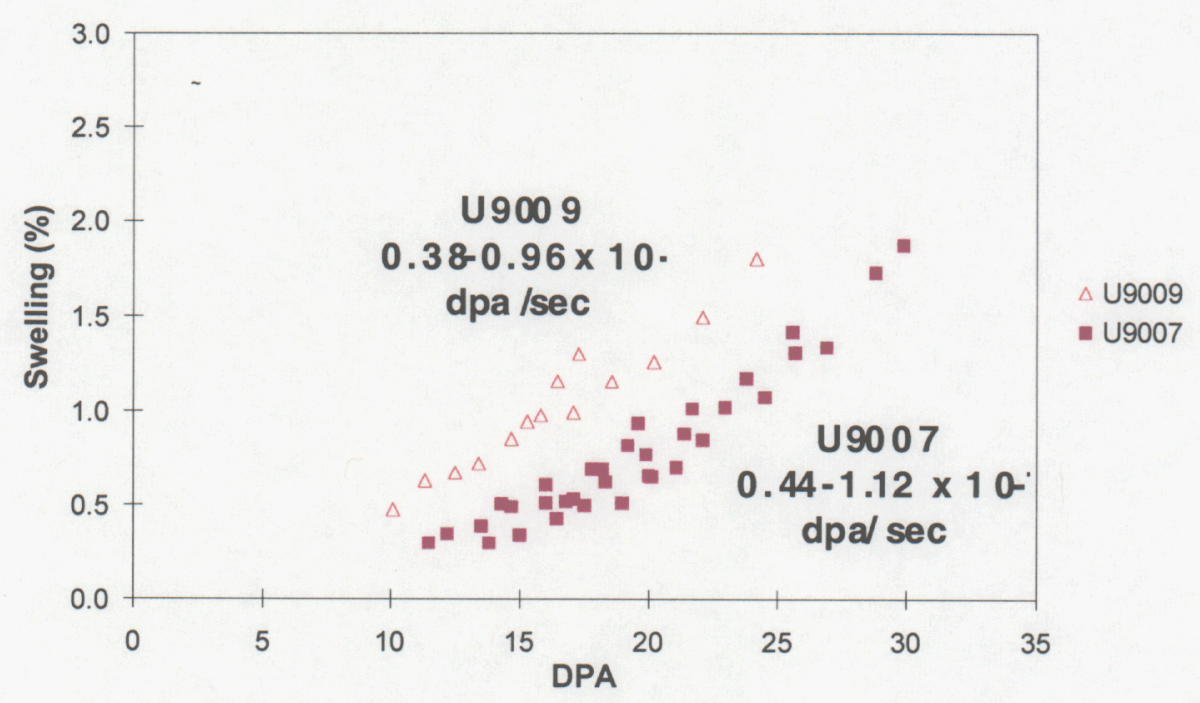

(b)

Figure 20. Swelling of annealed 304 stainless is strongly dependent on dpa rate at a given irradiation temperature. Note that each data set spans a range of dpa rates. The lowest dpa levels correspond to the lowest dpa rates. a) At temperatures of $373-388^{\circ} \mathrm{C}$ the transient regime of swelling is progressively decreased when moving from EBR-II row 8 to row 14 . b) When comparing two row 10 assemblies with dpa rates varying only $\sim 16 \%$, the strong effect of dpa rate can still be observed.

Figure 20 shows a subset of the swelling data. All data in the temperature range $373-388^{\circ} \mathrm{C}$ are plotted vs. dpa. Note that there are relatively small variations in dpa rate of the data from a given subassembly in this temperature subset, but the data clearly show that the transient regime of 
swelling is progressively shortened as the dpa rate decreases, such that only $10 \mathrm{dpa}$ are required to reach $1 \%$ swelling in row 14 . In previous publications it was shown that $30-50$ dpa were required to exceed $1 \%$ when data were collected at these temperatures from rows $2-4$ inside the EBR-II core at higher dpa rates.

The larger data of 280 data confirms the general validity of the effect of decreasing dpa rate to strongly increase swelling at all examined temperatures by shortening the duration of the transient regime. At temperatures above $388^{\circ} \mathrm{C}$ the impact of dpa rate was found to be even more pronounced than shown in Figure 21.

- Unexpectedly large amounts of helium and hydrogen are found in pure nickel irradiated to high fluence at high temperature.

Hydrogen and helium measurements in pure nickel irradiated to 100 dpa in HFIR at temperatures between 300 and $600^{\circ} \mathrm{C}$ show higher gas concentrations than predicted from fast-neutron reactions and the two-step ${ }^{58} \mathrm{Ni}(\mathrm{n}, \gamma)^{59} \mathrm{Ni}(\mathrm{n}, \mathrm{p}$ and $\mathrm{n}, \alpha)$ reactions. This additional gas production suggests previously unidentified nuclear sources of helium and possibly hydrogen that assert themselves at very high neutron exposure. Earlier helium measurements in the High Flux Isotopes Reactor (HFIR) appeared to be in good agreement with calculations and neutron dosimetry measurements up to thermal neutron fluences of about $4 \times 10^{22} \mathrm{n} / \mathrm{cm}^{2}$. New helium measurements at much higher thermal neutron fluences up to $1.1 \times 10^{23} \mathrm{n} / \mathrm{cm}^{2}$, as detailed below, show an excess of helium over the predictions. Furthermore, measurements using a new hydrogen system developed recently at PNNL, have shown that pure nickel samples irradiated to the same high fluences also have more hydrogen than would be predicted. These elevated hydrogen measurements are especially surprising since hydrogen is thought to be very mobile at the temperatures of the HFIR experiments so that the hydrogen would diffuse out of the samples, never reaching the levels observed in our measurements.

All of the samples used for the hydrogen and helium measurements were high purity (99.99+\%) nickel wires used for neutron dosimetry in various HFIR materials irradiation experiments for US and Japanese fusion reactor materials programs. Consequently, the neutron exposures are very well known for all of the samples used to measure helium and hydrogen that are cited in this paper. Thermal neutron fluences have an uncertainty of about 5-6\% and fast neutron fluences are known to about $10 \%$.

All of the helium measurements for pure nickel samples irradiated in HFIR are shown as a function of the thermal neutron fluence in (Figure 21). The measured helium values range up to about 35,000 appm or about 3.5 at\%. The stainless steel dpa values are shown near each helium measurement since stainless steel dpa is typically used as an exposure parameter for the HFIR irradiations. The dpa values for the pure nickel samples would be considerably higher, up to about $100 \mathrm{dpa}$, due mainly to the extra dpa from the high energy recoil from the ${ }^{59} \mathrm{Ni}(\mathrm{n}, \alpha)$ reaction. The $\mathrm{Ni}$ dpa values are 59, 78, and 104 for the corresponding stainless steel values of 34,44 , and 59 dpa shown. The line in Figure 21 represents the standard calculation of helium from nickel. 


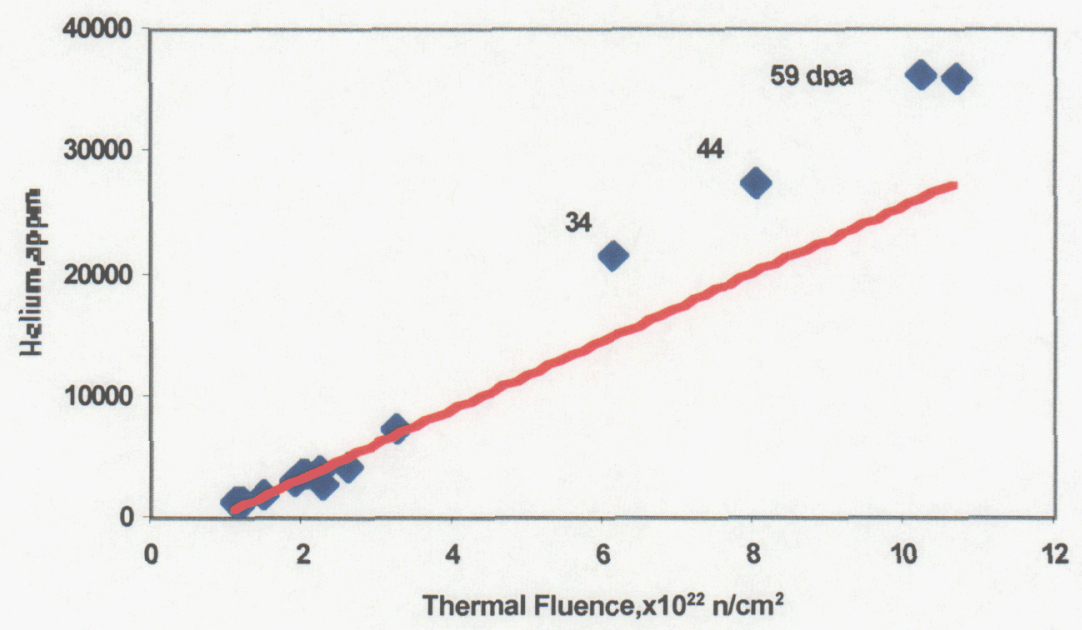

Fig. 21. Measured and calculated helium production from nickel irradiated in HFIR. The solid line is calculated using the evaluated ${ }^{59} \mathrm{Ni}$ cross sections.

The hydrogen measurements for pure nickel irradiated in HFIR are shown in (Figure 22). The diamonds show the measured values while the circles show the calculations using the standard model. The dpa values shown on the figure again refer to stainless steel rather than nickel since the HFIR experiments involved mainly stainless steel samples. As can be seen, the measured hydrogen values exceed the predicted values in most cases over the range of temperatures from 300 to $600^{\circ} \mathrm{C}$. It should be noted that the excess hydrogen seen in most of the samples could be due to environmental sources rather than simply nuclear production. The HFIR samples are not in contact with water, which is known to produce copious amounts of hydrogen through radiolysis in water reactors. However, at the elevated temperatures of the HFIR experiments, hydrogen is very mobile so that the samples have a flux of hydrogen moving through them during the experiment. The main point is that some of this hydrogen must be retained at the end of the experiment, contrary to our usual expectations. It should also be mentioned that the exact temperature values are not known since the dosimetry capsules were located between experimental capsules that had temperature monitoring. The dosimeters are believed to have temperatures between the values of the adjacent experimental assemblies, although exact values are not available. 


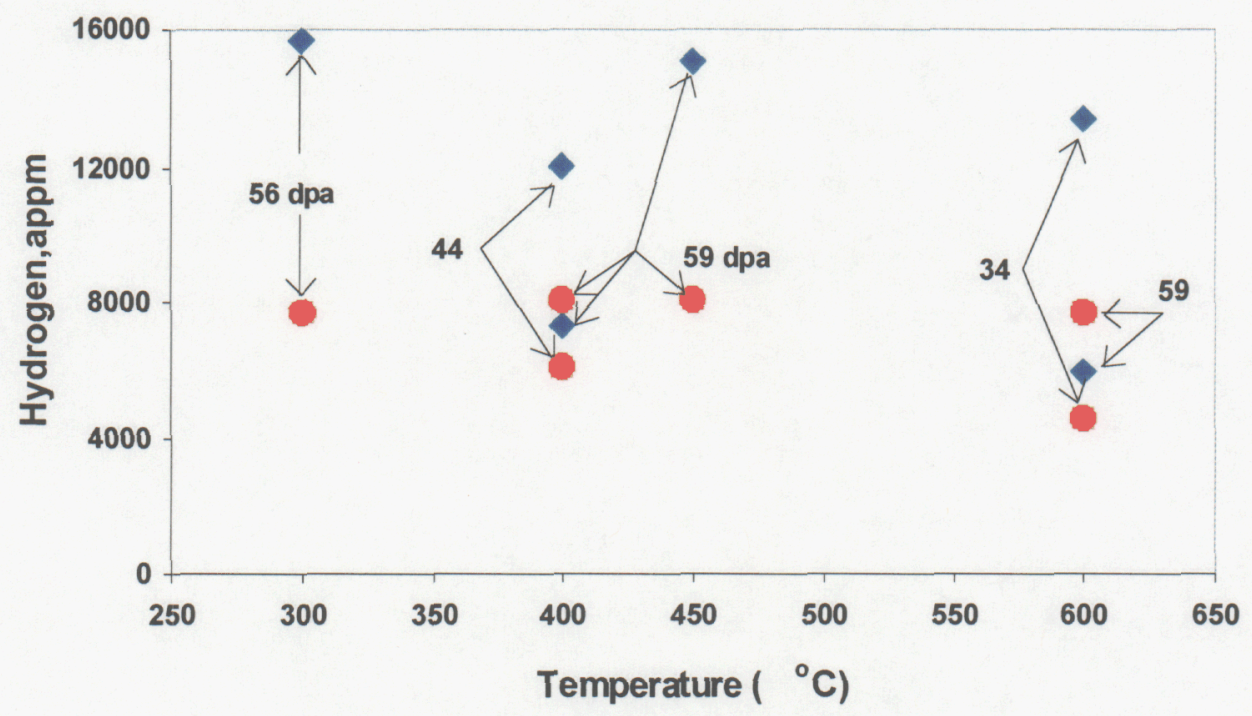

Fig. 22. Measured (diamonds) and calculated (dots) hydrogen in nickel irradiated in HFIR to various $316 \mathrm{SS}$ dpa values (arrows) as a function of irradiation temperature.

Figures 21 and 22 show the calculated production of helium and hydrogen in pure nickel from both the thermal neutron reactions for ${ }^{59} \mathrm{Ni}$ as well as the transmutation between the nickel isotopes for the fast neutron gas production. As can be seen, both the helium and the hydrogen measurements are significantly higher than the calculations at the higher neutron fluences. As mentioned above, the net transmutation to higher mass nickel isotopes with increasing neutron exposure has little effect on the net gas production since the various nickel isotopes have similar cross sections and, more importantly, gas production is dominated by the ${ }^{59} \mathrm{Ni}$ reactions. Since the excess hydrogen is most likely due to environmental sources, only the excess helium production needs to be explained.

The uncertainties in the ${ }^{59} \mathrm{Ni}$ cross sections listed cannot explain the differences seen between the measured and calculated helium data, especially in light of new ${ }^{59} \mathrm{Ni}$ measurements.

Uncertainties in the neutron fluences determined by the reactor dosimetry measurements are also far too small to explain the excess helium production; especially since all of the HFIR data have the same systematic sources of uncertainty. The net uncertainty on the ${ }^{59} \mathrm{Ni}$ content of the samples is estimated to be only $1 \%$. The most probable explanation for the excess helium production at high neutron fluences is that a daughter or granddaughter isotope produced by transmutation from natural nickel may produce helium.

\section{Planned Activities}

During the initial stage of dislocation evolution in solution-annealed stainless steels, a high density of small dislocation loops are present. While these loops merge with the network dislocations at higher doses, they are expected to influence the void nucleation, and hence the incubation dose for void swelling.

We plan to develop appropriate models for loop evolution during FY02 and incorporate them in the microstuctural evolution code.

Additional algorithm development will be done to improve the numerical stability of the code and thereby improve the efficiency.

Collision cascades lead to atomic mixing in materials with small precipitates. An analogous effect should lead to the destruction of small void embryos. This should result in an increase of 
the incubation dose for void swelling with increasing neutron energy. To quantify this potential effect of the neutron energy spectrum on incubation, collision cascade simulations will be carried near small clusters of vacancies with and without helium.

\section{Project Objectives Assessment}

A detailed status report on the entire project was given in the progress report that combined work performed during the second and third quarters. Little activity occurred during the first quarter at LLNL and UC Berkeley due to reduced funding for the first year.

In spite of the delayed start of the project, however, significant progress has been made. In particular, progress for the main task, namely the development of a microstuctural evolution code and the prediction of void swelling has exceeded my expectation. The code is fully operational, and prediction of void swelling for austenitic stainless steels are ahead of schedule. We have taken a departure from the original plan and developed a separate code for helium bubble formation. We have found that helium bubble formation in high nickel steels irradiated in thermal reactors precedes void formation. It is therefore advantageous to have a computational tool to predict property changes due to helium bubbles for situations where the total dose never exceeds the incubation dose for void swelling. Again, our modeling and analysis of helium bubble formation in structural materials is ahead of schedule, even though it has taken a different path than originally planned.

The task to be carried out by Prof. Chrzan and his students has only recently begun. This is somewhat behind schedule. It took time to find a good student. Besides, much research at universities is carried out during the summers.

\section{$\underline{\text { University Student Participation }}$}

Two students are participating on Prof. Daryl Chrzan's project, Chung-wei Yuan, who will carry out his Ph.D. research on the NERI project, and Mathew Sherburne who is a research assistant. We have presently a summer student working with us at LLNL. This graduate student, Dave Clatterbuck, is also from the Materials Science Department of UC Berkeley, and he is involved in modeling mechanical properties of materials. Prof. W. Morris is his advisor. All three students have US citizenship.

\section{Disclaimer:}

This document was prepared as an account of work sponsored by an agency of the United States Government. Neither the United States Government nor the University of California nor any of their employees, makes any warranty, express or implied, or assumes any legal liability or responsibility for the accuracy, completeness, or usefulness of any information, apparatus, product, or process disclosed, or represents that its use would not infringe privately owned rights. Reference herein to any specific commercial product, process, or service by trade name, trademark, manufacturer, or otherwise, does not necessarily constitute or imply its endorsement, recommendation, or favoring by the United States Government or the University of California. The views and opinions of the authors expressed herein do not necessarily state or reflect those of the United States Government or the University of California, and shall not be used for advertising or product endorsement purposes. 


\section{Auspices:}

This work was performed under the auspices of the U.S. Department of Energy by the University of California, Lawrence Livermore National Laboratory under Contract No. W-7405-Eng-48. This research was funded by the Department of Energy's Nuclear Energy Research Initiative (NERI) Program through the Office of Nuclear Energy, Science, and Technology and the Oakland Operations Office. 